Check for updates

Cite this: RSC Adv., 2017, 7, 25560

Received 20th February 2017

Accepted 18th April 2017

DOI: 10.1039/c7ra02111a

rsc.li/rsc-advances

\section{Fabrication of robust, superhydrophobic, electrically conductive and UV-blocking fabrics via layer-by-layer assembly of carbon nanotubes $\uparrow$}

\begin{abstract}
Xiao-Jing Guo, ${ }^{a}$ Chao-Hua Xue, (D) *ab Min Li, ${ }^{b}$ Xing Lic and Jian-Zhong Ma*ab
Multifunctional fabrics with excellent water repellency are very useful for practical applications. In this study, poly(ethylene terephthalate) (PET) fabrics were functionalized by introducing carbon nanotubes (CNTs) onto the PET fibers via a facile layer-by-layer electrostatic assembly using poly(dimethyl diallyl ammonium chloride) as a polyelectrolyte, followed by post-treatment with poly(dimethylsiloxane) (PDMS). The obtained fabric surfaces possessed superhydrophobicity, UV blocking properties and electrical conductivity. The hydrophobicity and electrical conductivities of the PET fabrics were systematically tunable by controlling the number of assembly layers. Wettability tests showed that the superhydrophobicity of the modified fabrics was robust to acid/alkaline etching, UV irradiation, long-time laundering and mechanical abrasion. Moreover, electrical conductivity showed no significant change even after repeating water laundering 20 times.
\end{abstract}

\section{Introduction}

In nature, many plants leaves, ${ }^{\mathbf{1 , 2}}$ insect legs, ${ }^{3}$ wings, ${ }^{\mathbf{4}}$ and animal feathers ${ }^{5}$ show extremely high water repellency, with a CA greater than $150^{\circ}$ and water sliding angle typically lower than $10^{\circ}$. Such materials are referred to superhydrophobic surfaces, ${ }^{6-14}$ attracting a lot of attention during the last decade mainly because of their unique water repellent and self-cleaning properties. With the deepening research on superhydrophobic surfaces, flexible substrates such as textiles have recently attracted great interest owing to the abundance and inexpensiveness of the raw materials, easy large scale-area production, and potential applications. ${ }^{15}$ Utilizing the fibrous microstructured textures, many researchers have coated fibers with nanostructures and hydrophobized the substrate to prepare superhydrophobic fabrics. ${ }^{16-19}$ From the practical point of view, it is still a great challenge to sustain the superhydrophobicity against laundering and abrasion during application.

To date, numerous durable superhydrophobic fabrics have been prepared on different fabrics adopting various methods/ techniques. ${ }^{20-26}$ For instance, Zimmermann et al. ${ }^{27}$ grew a layer of silicone nanofilaments on fibers to construct a hierarchically

\footnotetext{
${ }^{a}$ College of Environmental Science and Engineering, Shaanxi University of Science and Technology, Xi'an 710021, China. E-mail: xuech@zju.edu.cn; majz@sust.edu.cn

${ }^{b}$ College of Bioresources Chemistry and Materials Engineering, Shaanxi University of Science and Technology, Xi'an 710021, China

${ }^{c}$ College of Chemistry and Chemical Engineering, Shaanxi University of Science and Technology, Xi'an 710021, China
}

$\dagger$ Electronic supplementary information (ESI) available. See DOI: 10.1039/c7ra02111a rough structure to obtain superhydrophobic cotton fabrics. The coated fabric retained its superhydrophobic character even after continuous rubbing with a skin simulating friction partner under a force of $5 \mathrm{~N}$, making the nanofilaments wear off from the contact surfaces but remain intact elsewhere. Deng et al. ${ }^{28}$ prepared a laundry durable superhydrophobic cotton fabric using a radiation-induced graft polymerization method and the sample could withstand at least 100 commercial laundering cycles. Yoo et al. ${ }^{29}$ deposited a poly(1,3,5,7-tetravinyl-1,3,5,7tetramethylcyclotetrasiloxane) layer and a poly $(1 H, 1 H, 2 H, 2 H$ perfluorodecylacrylate) layer by initiated chemical vapor deposition, that allowed conformal coating of the stacked polymer film on micro-structured fibers. The fabric maintained its water repellency even after 20000 cycles of abrasion under a pressure of $9 \mathrm{kPa}$ and after 75 cycles of laundry. Our group has reported that fibers could be roughened through chemical etching at the surface, and superdurable superhydrophobic fabrics could be prepared by following diffusion of fluoroalkylsilane into the fibers $^{30}$ or forming covalent bonds between fibers and lowsurface-energy compounds. ${ }^{31,32}$ Despite the progress in the field, most of the methods still require harsh conditions, specific equipment, and fluorochemicals. It has been recognized that fluorochemicals have potential risks to human health and the environment. Meanwhile, it is noteworthy that there are few reports about simultaneously endowing fabric multifunction, such as electrical, UV-blocking and superhydrophobic properties.

For the past few years, layer-by-layer (LBL) assembly technique has been regarded as one of the most popular methods to fabricate functional composite coatings. ${ }^{33,34}$ LBL assembly involves alternate adsorption of species to form composite 
multilayer coating by electrostatic attractions, hydrogen-bonds, and coordination bonds, etc. ${ }^{33-39}$ The building species of LBL multilayer include polyelectrolytes, nano-particles and complexes of these materials. ${ }^{39,40}$ To fabricate multilayer coatings using polyelectrolytes, a charged substrate was alternately immersed into cationic and anionic aqueous solutions, then rinsed with water and dried in air. Hence, LBL assembly can be identified as a simple, inexpensive and versatile technique, which has been widely applied in preparation of various functional coatings. ${ }^{41,42}$

In this work, poly(dimethylsiloxane) (PDMS), carbon nanotubes (CNTs) and poly(dimethyl diallyl ammonium chloride) (PDDA) nanocomposites coating was constructed on poly(ethylene terephthalate) (PET) fabrics to simultaneously achieve electrical, UV-blocking and superhydrophobic properties via layer-by-layer assembly technique. The wettability tests showed that the superhydrophobic fabrics were robust to different chemicals and maintained superhydrophobicity after severe abrasion, and laundering, as well as long time exposure to UV irradiation. And the as-obtained fabrics showed excellent UVblocking property and electrical conductivity, which showed no great change even after repeated water laundering of 20 times.

\section{Experimental}

\section{Materials}

PDDA was purchased from Haining City Huangshang Chemical Industry Co., Ltd. PDMS, Sylgard 184 Silicone Elastomer Kit with components of PDMS base and curing agent, was purchased from DOW CORNING. PET fabric was purchased from a local factory. CNTs were purchased from Chengdu Organic Chemicals Co., Ltd. Chinese Academy of Science. All chemicals were used without further purification.

\section{Preparation of multifunctional coating on PET fabric by layer- by-layer self-assembly technique}

(1) PDMS precursor Part-A (Sylgard 186 elastomer base, 2 g) and PDMS precursor Part-B (Sylgard 186 curing agent, $0.2 \mathrm{~g}$ ) were dissolved in $100 \mathrm{~g}$ THF and magnetic stirred for $5 \mathrm{~min}$ at room temperature to form a coating solution. (2) $1 \mathrm{~g}$ PDDA was dissolved in $200 \mathrm{~g}$ deionized water and magnetic stirred for $5 \mathrm{~min}$ at room temperature. (3) $400 \mathrm{mg}$ CNTs and $200 \mathrm{~g}$ THF were mixed together and ultrasonicated for $20 \mathrm{~min}$ to form a stable CNT dispersion solution. (4) PET fabric was firstly immersed into PDDA solution for $3 \mathrm{~min}$ and dried at $80{ }^{\circ} \mathrm{C}$. Then the PDDAcoated fabric was immersed into CNTs dispersion solution for $5 \mathrm{~min}$ and dried at $80^{\circ} \mathrm{C}$. By repeating this process in a cyclic manner, a multilayer of $(\mathrm{CNT} / \mathrm{PDDA})_{n}$-coated fabric was obtained, where $n$ represents the cycle number. To give the fabric superhydrophobicity, the (CNT/PDDA $)_{n}$-coated fabric was finally immersed into PDMS solution for $10 \mathrm{~min}$ and dried at $80^{\circ} \mathrm{C}$. The obtained samples were denoted as PDMS/(CNT/PDDA $)_{n}$.

\section{Characterization}

SEM images were obtained under a Hitachi S-4800 field emission scanning electron microscope. Contact angle (CA) measurements were performed with a video optical contact angle system (OCA 20, Data physics, Germany) at ambient temperature with a $5 \mu \mathrm{L}$ water droplet as the indicator. All the CAs were determined by averaging values measured at 6 different points on each sample surface. TGA data were obtained using NETZSCH STA $409 \mathrm{PC} / \mathrm{PG}$ at a heating rate of $10{ }^{\circ} \mathrm{C}$ $\min ^{-1}$ in an $\mathrm{N}_{2}$ atmosphere. The reflectance and transmittance of the textiles were measured on an Agilent Cary 5000 UV-visNIR spectrophotometer in the range of 200 to $800 \mathrm{~nm}$. Electrical resistance measurements were performed on an ST-2258C multifunction digital four-probe tester. The electrical resistance was measured 5 times on each fabric.

\section{Chemical stability}

Aqueous solution with different $\mathrm{pH}$ values were used to test the chemical stability of superhydrophobic PET fabric. The samples were immersed into the selected solutions for $72 \mathrm{~h}$, rinsed with deionized water and dried at $80{ }^{\circ} \mathrm{C}$.

\section{Abrasion stability}

Abrasion durability tests were performed according to a modified procedure 29-31 based on the AATCC Test Method 8-2001. The pristine PET fabric was used as the abrasion partner. During the test, $45 \mathrm{kPa}$ of loading pressure was applied, and the sample was fixed onto a dynamic stainless steel column and moved repeatedly with a distance of $20 \mathrm{~cm}$ for one cycle.

\section{Washing durability}

The washing durability was evaluated by reference of the washing procedure described in the AATCC Test Method 612003 test no. 1A. The test was performed using a standard laundering machine (SW-12 E, Fang Yuan, China) at $40{ }^{\circ} \mathrm{C}$ in the presence of 10 stainless steel balls and $0.37 \mathrm{wt} \%$ soap powder in the washing containers. One cycle $(45 \mathrm{~min})$ of this standard washing procedure is equivalent to five cycles of home machine launderings in this work.

\section{UV irradiation resistance}

The coated fabrics were subjected to a UV irradiation using an artificial light source (UV lamp, Osram Ultra Vitalux $300 \mathrm{~W}$ ) emitting a Gaussian-shaped spectrum which peaked at $370 \mathrm{~nm}$ with a cut off at $290 \mathrm{~nm}$. Fabrics were placed under the UV lamp for continuous irradiation.

\section{Results and discussion}

\section{Characterization of coated fabrics}

Layer-by-layer self-assembly method is an important strategy to modify inert substrates for further functionalization. It is generally known that conventional PET fiber surfaces have few functional chemical groups, showing little affinity for functional chemicals. As shown in Fig. 1, the pristine PET fabric was treated with PDDA solution firstly, making the fiber surfaces positively charged. Secondly, the PDDA coated fabrics were immersed into CNTs dispersion, and the fiber surfaces would 


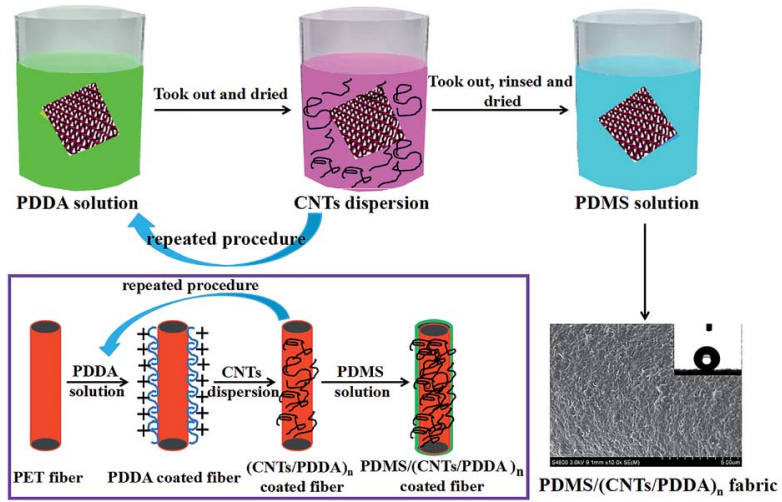

Fig. 1 Illustration of the fabrication of multifunctional superhydrophobic fabrics.

be covered by CNTs due to the electrostatic interaction between PDDA and CNTs. This procedure could be repeated for given times. Finally, the $(\mathrm{CNTs} / \mathrm{PDDA})_{n}$ coated fabrics were treated with $1 \%$ PDMS solution to render the fabrics superhydrophobicity.

The surface morphological evolution of the PET fibers was investigated by SEM at different magnifications, as shown in Fig. 2. SEM images show that the pristine PET fiber exhibits a round and smooth surface (Fig. 2(a) and (b)). After being assembled with 10 layers of CNT/PDDA, the fiber surface became much rougher than the pristine one (Fig. 2(c) and (d)), and uniformly covered by countless nano-scale CNTs, indicating that CNTs had been successfully connected to the PET
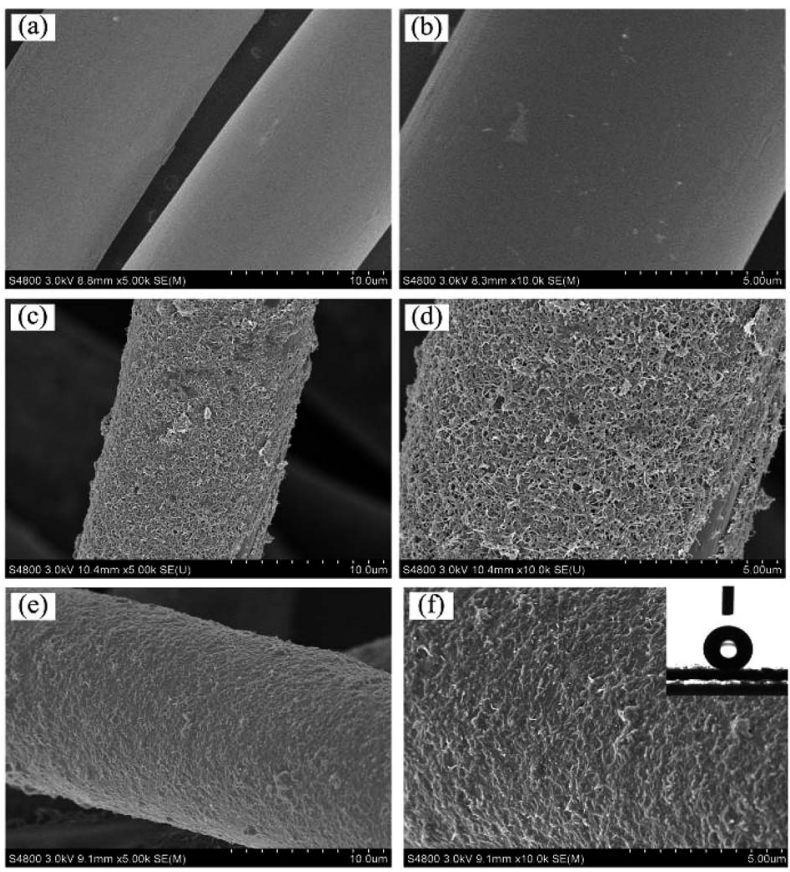

Fig. 2 SEM images of (a) pristine PET fabrics, (b) higher magnification of (a), (c) (CNTs/PDDA) 10 coated fabrics, and (d) higher magnification of (c), (e) PDMS/(CNTs/PDDA) 10 coated fabrics, and (f) higher magnification of $(c)$. The inset in (f) shows the CA of PDMS/(CNTs/PDDA) 10 coated fabrics. fabric. Treatment of the (CNTS/PDDA $)_{10}$ coated fabric with PDMS solution resulted in dense and uniform coating of fibers by polymers, as shown in Fig. 2(e and f). The micro-scale structure of PET fabric in combination with the nano-scale protrusions created a hierarchical rough structure, which is very similar to the structure that as observed in a lotus leaf. It is well known that a superhydrophobic surface generally has a low surface energy material combined with a particular micro/nano structural roughness. This Cassie's mode-like structure of the PDMS/(CNTs/PDDA $)_{10}$ PET fabric can effectively reduce the solid-liquid contact area, possessing a CA of $166.9^{\circ} \pm 1^{\circ}$ (inset of Fig. 2(d)).

The thermal decomposition of pristine PET fabric and PDMS/(NT/PDDA $)_{10}$ coated PET fabric were studied by thermo gravimetric analysis in nitrogen atmosphere, as shown in Fig. 3. It can be seen from the curves that the pristine PET fabric went through two weight losses, and the sharp weight loss between $400-460{ }^{\circ} \mathrm{C}$ corresponds to the oxidative decomposition of the PET backbone. As the temperature reached $600{ }^{\circ} \mathrm{C}$, the pristine PET fabric completely lost its weight of $89 \%$. As for PDMS/(CNT/ PDDA $)_{10}$ coated PET fabric, the residue amount increased to $21 \%$ at $600{ }^{\circ} \mathrm{C}$ indicating that the treated PET fabric shows higher stability than pristine PET. This is attributed to the good thermal stability of CNT, as seen from the weight loss curve of CNTs in Fig. 3.

\section{Hydrophobic properties of the coated fabrics}

Surface wettability examination showed that water can easily spread on the pristine PET fabric due to the capillary effects of the fibrous structure, as shown in Fig. 4(a). To give the fabric superhydrophobicity, the (CNT/PDDA $)_{n}$-coated fabric was immersed into PDMS solution. The hydrophobicity of the surfaces can be tuned by controlling the assembly layers of CNTs, as shown in Fig. 4(c). After being coated with (CNT/ PDDA $)_{n}$ multilayers and further treated with PDMS, the fabrics became superhydrophobic. Increasing the layers of PDDA/CNTs increased the CAs and decreased the SAs correspondingly, which finally converted the hydrophilic fabrics to superhydrophobic ones (Fig. 4(b)) with a CA of $166.9^{\circ} \pm 1^{\circ}$ and SA of $4.2^{\circ} \pm 1.5^{\circ}$, making water droplets roll easily.

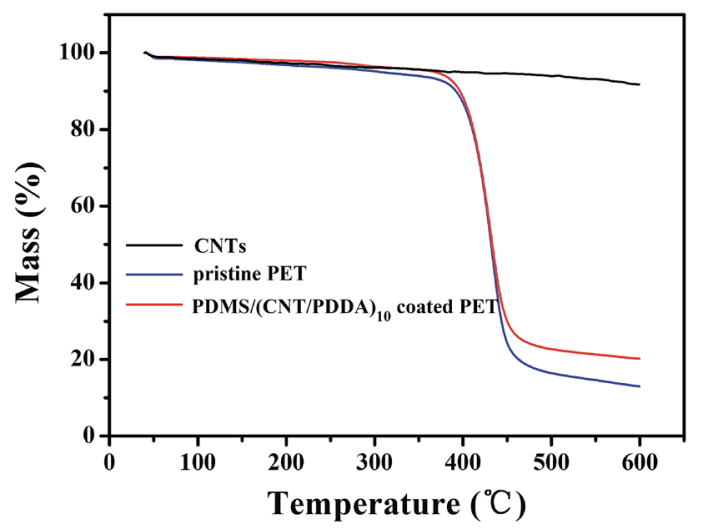

Fig. 3 TGA curves of pristine PET, PDMS/(CNT/PDDA) 10 coated PET fabrics and CNT. 


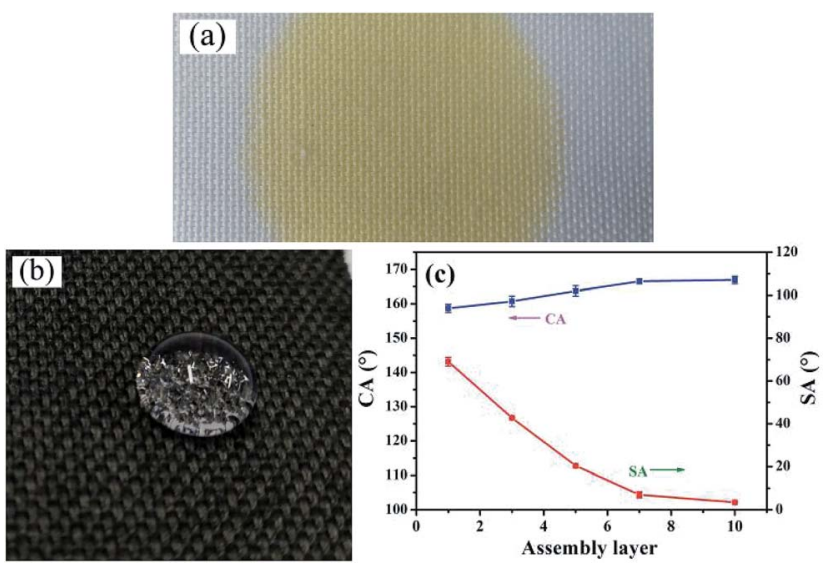

Fig. 4 (a) Digital images of dyed water droplets after contact with the (a) pristine and (b) PDMS/(CNTs/PDDA) 10 PET fabrics. (c) The relationship between the CA/SA of modified fabrics and number of assembly layers.

\section{Stability of the superhydrophobic fabrics}

For fabrics, good laundering durability is of significance for reuse. To check the robustness of the superhydrophobicity of the coated samples against laundry processes, the fabric was put into a commercial laundry machine and made undergo washing cycles with $0.37 \%$ of detergent and ten stainless steel balls added. Such a mechanical agitation, in conjunction with various chemicals in the detergent, offers an extremely harsh environment for superhydrophobic fabrics. Fig. 5(b) shows the changes of CAs and SAs, monitored according to the number of laundry cycles. The test was performed for 20 cycles, approximate to 100 cycles of commercial laundering. The CA was decreased but still higher than $155^{\circ}$. However, the SA increased from $4.2^{\circ} \pm 1.5^{\circ}$ to $13.5^{\circ} \pm 1.5^{\circ}$. There are two reasons for this phenomenon. On one hand, as laundering cycles increased, the roughness on the coated fiber surface decreased due to the mechanical action. On the other hand, with severe mechanically laundering, some protruding fuzzes appeared on the surface of the sample, causing some force or adhesion to water on the fabric. Thus, the SA increased with increasing the laundering cycles.

The abrasion durability was evaluated by a modified procedure based on the AATCC Test Method 8-2001 using untreated PET fabric as abrasion partner. During the test, $45 \mathrm{kPa}$ of pressure were employed. Fig. 6(d) shows the change of the CA with the abrasion cycles. It was found that the CA of the modified
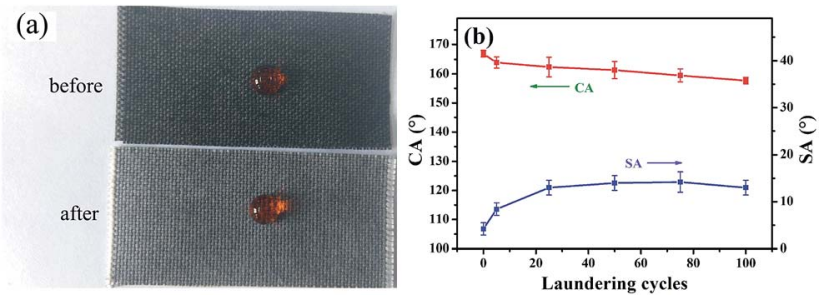

Fig. 5 (a) Photograph of a water droplet on the PDMS/(CNT/PDDA) 10 fabrics before and after laundering test, (b) changes of CA and SA of PDMS/(CNT/PDDA $)_{10}$ fabrics with washing cycles.

fabrics decreased from $166.9^{\circ} \pm 1^{\circ}$ to $160.5^{\circ} \pm 2^{\circ}$ after 2000 abrasion cycles, and the coated fabric can withstand at least 10000 cycles of abrasion damages without changing its superrepellent feature, indicating excellent durability of superhydrophobicity against abrasion, although the samples were worn out (Fig. 6(a)). As for the SA, it increased from $4.2^{\circ} \pm 1.5^{\circ}$ to $16.5^{\circ} \pm 1.2^{\circ}$, which is due to the partial removal of the coating at the fiber surface and lowering of fiber roughness (Fig. 6(b)).

Apart from abrasion and laundering test, we also evaluated the durability of superhydrophobicity of PDMS/(CNT/PDDA $)_{10}$ fabrics by irradiating the samples under a $300 \mathrm{~W}$ UV-lamp or immersing into solutions with different $\mathrm{pH}$ for 72 h. Fig. 7(a) shows the CA and SA of PDMS/(CNT/PDDA $)_{10}$ fabrics as a function of UV irradiation time. The results showed no significant change after different UV exposure time. Fig. 7(b) presents that the CAs of the samples were nearly unchanged, indicating their strong resistance to different $\mathrm{pH}$ solutions.

\section{Electrical properties of the PDMS/(CNTs/PDDA $)_{10}$ coated PET fabrics}

Carbon nanotubes (CNTs) have been attracting much attention due to their excellent electrical, mechanical, and thermal
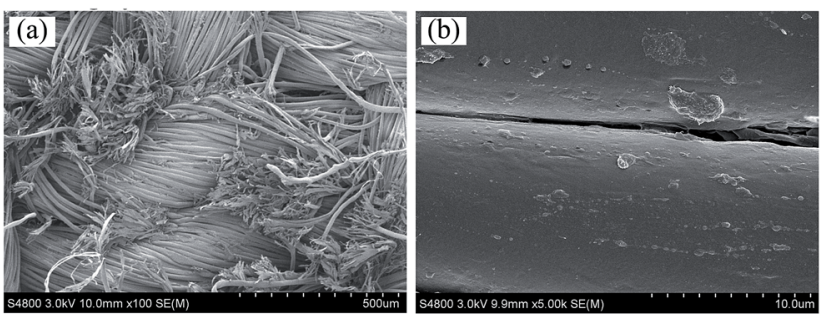

(c)
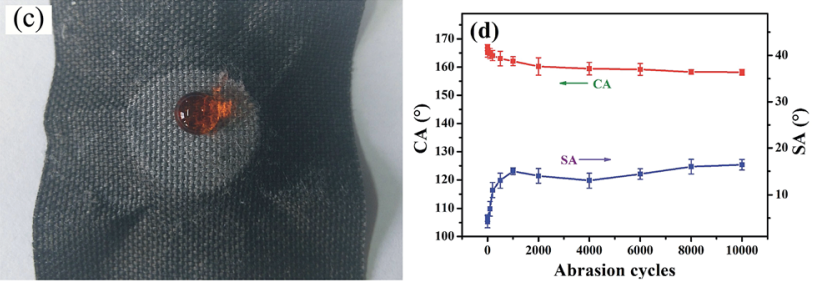

Fig. 6 (a) SEM image of PDMS/(CNT/PDDA) $)_{10}$ fabrics after abrasion test of 10000 cycles. (b) Higher magnification of (a). (c) Photograph of a water droplet on the PDMS/(CNT/PDDA) 10 fabrics after 10000 cycles. (d) Changes of CA and SA of PDMS/(CNT/PDDA) 10 fabrics with abrasion cycles.
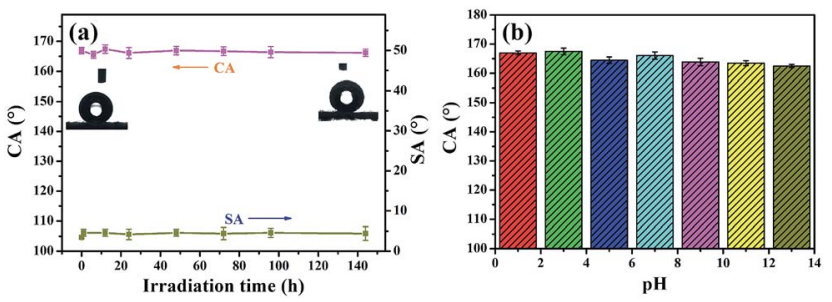

Fig. $7 \mathrm{CA}$ and SA changes of PDMS/(CNT/PDDA) 10 fabrics with UV irradiation time, (b) CA of PDMS/(CNT/PDDA) 10 fabrics treated by (a) immersion in different $\mathrm{pH}$ solutions for $72 \mathrm{~h}$. 
properties. Among these properties, the extremely high conductivity and large aspect ratio are very effective as a filler in conductive composite materials. It should be noted that pristine PET fabrics are not conductive, showing very high resistivity. However, seven times coating of CNTs could make the fabric conductive, and stable conductive fabrics could be obtained after ten times assembling, with a resistivity as low as $6.54 \Omega \mathrm{cm}^{-1}$, as shown in Fig. 8(a). Importantly, further treatment of (CNTs/PDDA $)_{10}$ fabrics using polymer PDMS, did not cause much increase of the resistivity which maintained $37.5 \Omega$ $\mathrm{cm}^{-1}$, showing excellent electrical conductivity. This is because that PDMS coating did not disrupt the well connected conductive CNTs network on the PDMS/(CNTs/PDDA $)_{10}$, fabric could easily help to power on an LED device connected to a battery (Fig. 8(b)). And commercial laundering of 20 cycles only increased the electrical resistivity from 37.5 to $57.3 \Omega \mathrm{cm}^{-1}$. It indicates that the fabrics possess excellent durability of conductivity against water laundering.

\section{Optical properties of the PDMS/(CNTs/PDDA $)_{n}$ coated PET fabrics}

Generally, the optical performance of materials depends on its ability to reflect or absorb the light. As shown in Fig. 9(a), the colour of the fabrics changed from white to black gradually with increasing the assembled layers of CNTs. To examine the colour change effect on their optical properties, we checked the reflectance of the samples in the range of 200-800 $\mathrm{nm}$ and the results were shown in Fig. 9(b). The untreated fabric has a low reflectance at $200-300 \mathrm{~nm}$, but a higher one at $400-800 \mathrm{~nm}$, since the penetration of UV light is stronger than visible light. Meanwhile, the reflectance of corresponding modified fabrics were decreased with increasing assembled layers of CNTs. Fig. 9(c) showed the transmittance of the fabrics in the range of 200 to $800 \mathrm{~nm}$. For the CNTs coated PET fabrics, the transmission between $200-380 \mathrm{~nm}$, UV radiation region, significantly decreased compared to the pristine PET fabric. The strong absorbance of the PDMS/(CNTs/PDDA $)_{n}$ coated PET fabrics in the ultraviolet wavelengths may find great potential applications in photoprotection. The UV-blocking properties of modified PET fabric may be due to excellent adsorption of CNTs.

Actually, layer-by-layer assembly technique can be used to modify various kinds of substrates. Therefore, as a control experiment, the same experimental process was used to modify
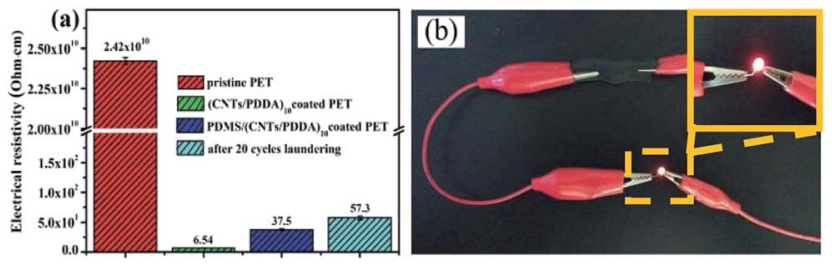

Fig. 8 (a) Electrical resistivity of pristine PET, $(C N T s / P D D A)_{10}$ coated $\mathrm{PET}, \mathrm{PDMS} /(\mathrm{CNTS} / \mathrm{PDDA})_{10}$ coated PET and after 20 cycles laundering of PDMS/(CNTs/PDDA) 10 coated PET fabrics. (b) Demonstration of LED emission with the current passing through PDMS/(CNTs/PDDA) ${ }_{10}$ coated PET fabrics.
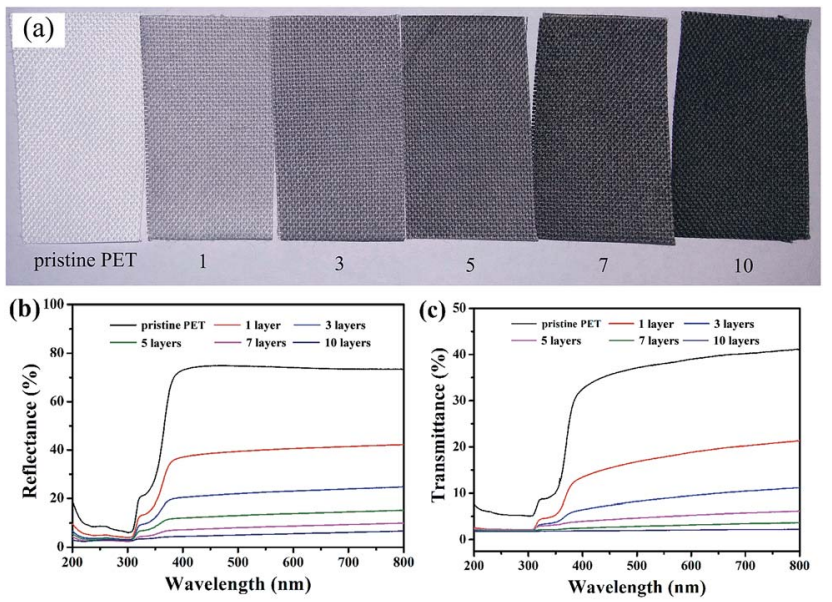

Fig. 9 (a) Photograph of pristine PET, PDMS/(CNT/PDDA) $n$ PET fabrics, in which the numbers mean the LBL layers; (b) UV-visible reflectance of pristine PET, PDMS/(CNT/PDDA) ${ }_{n}$ PET fabrics; (c) UVvisible transmittance of pristine PET, PDMS/(CNT/PDDA) ${ }_{n}$ PET fabrics.

cotton fabric and the corresponding results were shown in ESI Fig. S1-S4. $\dagger$ It was found that stable superhydrophobicity and electrically conductivity could be obtained after 7 times assembling. Compared with PET fabric, the cotton fabric was more difficult to obtain superhydrophobicity and easier to get electrically conductivity due to the abundant hydroxyl groups on the fiber surfaces, which enhance the affinity of CNTs/PDDA for the fibers. Anyway, the as-obtained fabrics exhibited superhydrophobic, electrically conductive and UV-blocking properties by the same method.

\section{Conclusions}

We have demonstrated that superhydrophobic, UV blocking properties and electrical conductive PET fabrics were successfully fabricated via layer-by-layer assembly of CNTs followed by coating with PDMS. The as-obtained fabrics possess remarkable durability against different $\mathrm{pH}$ solutions without changing its super-repellent feature and exhibit excellent resistance to washing, abrasion, even exposure to UV light. The electrical resistivity of $\mathrm{PDMS} /(\mathrm{CNT} / \mathrm{PDDA})_{10}$ PET fabrics reached at 37.5 $\Omega \mathrm{cm}$, which decrease approximate $10^{7}$ times compared with pristine PET fabrics. The water laundering durability tests indicated that the modified fabrics still performs efficient electrical conductivity after 20 cycles of commercial laundering. Moreover, the whole procedure was carried out in a mild environment, and no special instruments were needed. Utilizing the excellent UV absorption property and electrical conductivity, the superhydrophobic PDMS/(CNT/PDDA $)_{10}$ PET fabrics might find great potential applications for outdoor products.

\section{Acknowledgements}

This work was supported by National Natural Science Foundation of China (51372146, 51572161), Research Fund for the Doctoral Program of Higher Education of China 
(20136125110003), Major Program of Science Foundation of Shaanxi Province (2011ZKC05-7), Key Scientific Research Group of Shaanxi province (2013KCT-08), and Scientific Research Group of Shaanxi University of Science and Technology (TD12-03).

\section{Notes and references}

1 K. Koch, B. Bhushan and W. Barthlott, Prog. Mater. Sci., 2009, 54, 137-178.

2 N. J. Shirtcliffe, F. Brian Pyatt, M. I. Newton and G. McHale, J. Plant Physiol., 2006, 163, 1193-1197.

3 X. Gao and L. Jiang, Nature, 2004, 432, 36.

4 E. Bormashenko, Y. Bormashenko, T. Stein, G. Whyman and E. Bormashenko, J. Colloid Interface Sci., 2007, 311, 212-216.

5 W. Lee, M.-K. Jin, W.-C. Yoo and J.-K. Lee, Langmuir, 2004, 20, 7665-7669.

6 H. Bellanger, T. Darmanin, E. Taffin de Givenchy and F. Guittard, Chem. Rev., 2014, 114, 2694-2716.

7 J. Li, L. Li, X. Du, W. Feng, A. Welle, O. Trapp, M. Grunze, M. Hirtz and P. A. Levkin, Nano Lett., 2015, 15, 675-681.

8 Y. Wang, Y. Shi, L. Pan, M. Yang, L. Peng, S. Zong, Y. Shi and G. Yu, Nano Lett., 2014, 14, 4803-4809.

9 C.-H. Xue and J.-Z. Ma, J. Mater. Chem. A, 2013, 1, 4146-4161. 10 X. Du, J. S. Li, L. X. Li and P. A. Levkin, J. Mater. Chem. A, 2013, 1, 1026-1029.

11 X. Zhou, Z. Zhang, X. Xu, F. Guo, X. Zhu, X. Men and B. Ge, ACS Appl. Mater. Interfaces, 2013, 5, 7208-7214.

12 B. Wang, J. Li, G. Wang, W. Liang, Y. Zhang, L. Shi, Z. Guo and W. Liu, ACS Appl. Mater. Interfaces, 2013, 5, 1827-1839.

13 D. Goswami, S. K. Medda and G. De, ACS Appl. Mater. Interfaces, 2011, 3, 3440-3447.

14 P. S. Brown and B. Bhushan, Sci. Rep., 2015, 5, 8701.

15 F. Chen, H. Yang, X. Liu, D. Chen, X. Xiao, K. Liu, J. Li, F. Cheng, B. Dong, Y. Zhou, Z. Guo, Y. Qin, S. Wang and W. Xu, ACS Appl. Mater. Interfaces, 2016, 8, 5653-5660.

16 L. Wu, J. Zhang, B. Li, L. Fan, L. Li and A. Wang, J. Colloid Interface Sci., 2014, 432, 31-42.

17 Z.-Y. Deng, W. Wang, L.-H. Mao, C.-F. Wang and S. Chen, J. Mater. Chem. A, 2014, 2, 4178-4184.

18 X. Chao-Hua, J. Xue-Qing, Z. Jing, M. Jian-Zhong and J. ShunTian, Nanotechnology, 2015, 26, 335602.

19 L. Wu, J. Zhang, B. Li and A. Wang, J. Mater. Chem. B, 2013, 1, 4756-4763.

20 Y. Zhao, Z. Xu, X. Wang and T. Lin, Langmuir, 2012, 28, 63286335.
21 C.-H. Xue, Y.-R. Li, P. Zhang, J.-Z. Ma and S.-T. Jia, ACS Appl. Mater. Interfaces, 2014, 6, 10153-10161.

22 H. Wang, Y. Xue, J. Ding, L. Feng, X. Wang and T. Lin, Angew. Chem., Int. Ed., 2011, 50, 11433-11436.

23 Y. Liu, Y. Liu, H. Hu, Z. Liu, X. Pei, B. Yu, P. Yan and F. Zhou, J. Phys. Chem. C, 2015, 119, 7109-7114.

24 C.-H. Xue, Y.-R. Li, J.-L. Hou, L. Zhang, J.-Z. Ma and S.-T. Jia, J. Mater. Chem. A, 2015, 3, 10248-10253.

25 H. Zou, S. Lin, Y. Tu, G. Liu, J. Hu, F. Li, L. Miao, G. Zhang, H. Luo, F. Liu, C. Hou and M. Hu, J. Mater. Chem. A, 2013, 1, 11246-11260.

26 M. Wu, B. Ma, T. Pan, S. Chen and J. Sun, Adv. Funct. Mater., 2016, 26, 569-576.

27 J. Zimmermann, F. A. Reifler, G. Fortunato, L.-C. Gerhardt and S. Seeger, Adv. Funct. Mater., 2008, 18, 3662-3669.

28 B. Deng, R. Cai, Y. Yu, H. Jiang, C. Wang, J. Li, L. Li, M. Yu, J. Li, L. Xie, Q. Huang and C. Fan, Adv. Mater., 2010, 22, 5473-5477.

29 Y. Yoo, J. B. You, W. Choi and S. G. Im, Polym. Chem., 2013, 4, 1664-1671.

30 C.-H. Xue, P. Zhang, J.-Z. Ma, P.-T. Ji, Y.-R. Li and S.-T. Jia, Chem. Commun., 2013, 49, 3588-3590.

31 C.-H. Xue, X.-J. Guo, J.-Z. Ma and S.-T. Jia, ACS Appl. Mater. Interfaces, 2015, 7, 8251-8259.

32 C.-H. Xue, X.-J. Guo, M.-M. Zhang, J.-Z. Ma and S.-T. Jia, J. Mater. Chem. A, 2015, 3, 21797-21804.

33 X. Chen, F. Fang, X. Zhang, X. Ding, Y. Wang, L. Chen and X. Tian, $R S C A d v$., 2016, 6, 27669-27676.

34 C. Schlaich, L. Cuellar Camacho, L. Yu, K. Achazi, Q. Wei and R. Haag, ACS Appl. Mater. Interfaces, 2016, 8, 29117-29127.

35 G. Decher, Science, 1997, 277, 1232-1237.

36 B.-S. Kong, J. Geng and H.-T. Jung, Chem. Commun., 2009, 2174-2176.

37 D. Wang, J. Yang, X. Li, D. Geng, R. Li, M. Cai, T.-K. Sham and X. Sun, Energy Environ. Sci., 2013, 6, 2900-2906.

38 J. Zou and F. Kim, Nat. Commun., 2014, 5, 5254.

39 A. Finnemore, P. Cunha, T. Shean, S. Vignolini, S. Guldin, M. Oyen and U. Steiner, Nat. Commun., 2012, 3, 966.

40 G. B. Sukhorukov, E. Donath, H. Lichtenfeld, E. Knippel, M. Knippel, A. Budde and H. Möhwald, Colloids Surf., A, 1998, 137, 253-266.

41 X. Zhang, H. Chen and H. Zhang, Chem. Commun., 2007, 1395-1405.

42 F.-X. Xiao, M. Pagliaro, Y.-J. Xu and B. Liu, Chem. Soc. Rev., 2016, 45, 3088-3121. 